\title{
Expression of CIITA-related MHCII molecules in tumors linked to prognosis in hepatocellular carcinoma
}

\author{
XING-WANG XIE ${ }^{1 *}$, MING-HUI MEI ${ }^{3 *}$, WEI-JIA LIAO ${ }^{3}$, LI-HUA QIAN $^{2}$, XIN YU $^{4}$, RAN FEI $^{1}$, LI-LING QIN ${ }^{3}$, \\ HENG-HUI ZHANG ${ }^{1}$, JI-RUN PENG ${ }^{4}$, DAN-HUA SHEN ${ }^{2}$, LAI WEI ${ }^{1}$ and HONG-SONG CHEN ${ }^{1}$ \\ ${ }^{1}$ Hepatology Institute, ${ }^{2}$ Department of Pathology, Peking University People's Hospital, Beijing 100044; \\ ${ }^{3}$ Guilin Medicial University, Guangxi Province 541000; ${ }^{4}$ Center of Hepatobiliary Surgery, \\ Peking University People's Hospital, Beijing 100044, P.R. China
}

Received October 17, 2008; Accepted December 8, 2008

DOI: 10.3892/ijo_00000194

\begin{abstract}
The prognosis of hepatocellular carcinoma (HCC) after surgery is poor due to its high recurrence rate. In order to unfold the mechanism of different recurrent-free survival (RFS) times following resection, expression profiling of tumor tissues from 32 HCC patients with different RFS time were used to identify differential expression of individual genes and signaling pathway components correlated with RFS time. Quantitative RT-PCR, Western blotting, and immunohistochemistry were used to validate the expression of selected genes. Up-regulation of several immune related genes and pathways, especially HLA II-related antigen presenting pathways, significantly correlated with longer RFS time. The expression of MHCII molecules were found to be mainly located in either $\mathrm{CD} 8^{+}$cells or $\mathrm{CD} 45^{+}$cells, and their expression significantly correlated with the expression of CIITA (HLA II genes transactivator) in the tumor. The results suggest that the high expression level of CIITA and MHCII molecules in hepatocellular carcinoma tissue is an effective prognostic marker for longer RFS time in HCC.
\end{abstract}

\section{Introduction}

Hepatocellular carcinoma (HCC) is one of the most common cancers in the world. Its prevalence is especially high in China due to the high rate of hepatitis B virus (HBV) infection. Currently, curative resection is the best treatment for HCC patients, but a recurrence rate of $61.5 \%$ within 5 years following surgery represents a major obstacle in

Correspondence to: Dr Hong-Song Chen, Peking University Hepatology Institute, Peking University People's Hospital, Beijing 100044, P.R. China

E-mail: chenhongsong2999@163.com

${ }^{*}$ Contributed equally

Key words: hepatocellular carcinoma, recurrence, CIITA, MHC II genes, microarray, gene expression profile improving the prognosis of HCC patients (1). Therefore, a better understanding of the molecular mechanism of recurrence and its impact may help us to develop more effective prognosis makers or therapeutic strategies to improve the prognosis of HCC patients.

Gene expression profiling using microarray analysis can identify the molecular mechanisms involved in the recurrence and metastasis of HCC (2-5). Molecular mechanisms involved in the recurrence or metastasis of carcinomas have been identified, and predictive gene expression signatures developed have shown the ability to predict recurrence, metastasis, and prognosis of HCC. These studies have confirmed a correlation between gene expression patterns of primary tumor lesions and prognosis of HCC patients.

Our present study investigated gene expression differences between HCC patients with different recurrent-free survival (RFS) times; especially for HCC patients with a disease-free survival time greater than 3 years. We aimed to add new finding to the mechanism of post-surgery recurrence, and to identify treatment targets that could prolong the survival of HCC patients following surgery.

\section{Materials and methods}

Hepatocellular carcinoma specimens. HCC patients who received curative surgical resection were included in this study and all patients were histopathologically diagnosed with primary HCC. Tumor tissue and adjacent non-tumor tissue were collected and snap-frozen in liquid nitrogen immediately following removal from patients. Informed consent was obtained from all participating patients.

Serum $\alpha$ fetoprotein (AFP) tests and abdominal ultrasonography (US) were used to identify tumor recurrence in follow-up clinical evaluations of patients. Catscans (CT) or magnetic resonance imaging (MRI) were performed if recurrence was suspected due to an abnormal AFP test or US examination. Follow-up for patients lasted until recurrence was diagnosed, or for at least 3 years following surgery. Patients who died of causes unrelated to HCC recurrence were excluded from this study. Follow-up data were completed for $32 \mathrm{HCC}$ patients with an RFS time that varied from 3 months to $>3$ years. These patients were divided into 
Table I. Summary of clinical characteristics of HCC patients.

\begin{tabular}{|c|c|c|c|c|}
\hline Patient characteristics & $\mathrm{S}$ & M & $\mathrm{L}$ & p-value \\
\hline Gender & & & & 0.401 \\
\hline Male & 9 & 8 & 10 & \\
\hline Female & 2 & 0 & 3 & \\
\hline Age (years) & $50.8 \pm 11.9$ & $62.6 \pm 7.6$ & $50.8 \pm 14.4$ & 0.076 \\
\hline Viral infection & & & & 0.748 \\
\hline HBV & 9 & 6 & 10 & \\
\hline $\mathrm{HCV}$ & 0 & 1 & 0 & \\
\hline- & 2 & 1 & 3 & \\
\hline AFP & & & & 0.486 \\
\hline$<20 \mathrm{ng} / \mathrm{ml}$ & 6 & 2 & 5 & \\
\hline $20 \mathrm{ng} / \mathrm{ml}$ & 5 & 6 & 8 & \\
\hline ALT & & & & 0.106 \\
\hline$\leq 40 \mathrm{U} / 1$ & 5 & 4 & 11 & \\
\hline$>40 \mathrm{U} / 1$ & 6 & 4 & 2 & \\
\hline AST & & & & 0.053 \\
\hline$\leq 40 \mathrm{U} / 1$ & 3 & 4 & 10 & \\
\hline$>40 \mathrm{U} / 1$ & 8 & 4 & 3 & \\
\hline Total bilirubin $(\mu \mathrm{m} / \mathrm{l})$ & $18.6 \pm 20.0$ & $14.5 \pm 8.5$ & $11.3 \pm 2.9$ & 0.375 \\
\hline Tumor size (cm) & $8.3 \pm 3.7$ & $7.4 \pm 2.7$ & $5.4 \pm 3.3$ & 0.109 \\
\hline TNM stage & & & & $0.004^{\mathrm{a}}$ \\
\hline $\mathrm{I}+\mathrm{II}$ & 3 & 8 & 7 & \\
\hline III + IV & 8 & 0 & 6 & \\
\hline Cirrhosis & & & & 0.420 \\
\hline No & 4 & 1 & 2 & \\
\hline Yes & 7 & 7 & 11 & \\
\hline Satellite nodules & & & & 0.104 \\
\hline No & 5 & 7 & 10 & \\
\hline Yes & 6 & 1 & 3 & \\
\hline
\end{tabular}

${ }^{\mathrm{a}} \mathrm{P}<0.01$.

three groups: patients with an RFS time of $<1$ year were labeled as having a 'short' RFS time and were assigned to an $\mathrm{S}$ group; patients with an RFS time between 1 and 3 years were labeled as having a 'middle' RFS time and were assigned to an $\mathrm{M}$ group; and patients with an RFS time of $>3$ years were labeled as having a 'long' RFS time and were assigned to an L group. The clinical characteristics of these patients are listed in Table I.

RNA preparation, amplification and labeling. Total RNA was extracted from each sample using TRIzol (Invitrogen, Carlsbad, CA) according to the manufacturer's instructions. NucleoSpin ${ }^{\circledR}$ RNA clean-up Kit (Macherey-Nagel, Germany) was used to purify the total RNA. RNA ampli-fication, cDNA labeling, hybridization, and scanning were performed according to methods described in a previous study (6). Briefly, a T7-oligodT primer was used to synthesize double stranded cDNA from $5 \mu \mathrm{g}$ total RNA to generate cRNA. Two micrograms of cRNA was reverse-transcribed into
cDNA, which was labeled with Cy5 and Cy3 using a Klenow enzyme reaction. Similarly, RNA from a mixed pool of adjacent non-tumor tissue derived from 9 patients was amplified, labeled, and used as a reference pool for all microarray hybridizations.

\section{Microarray analysis}

Oligonucleotide hybridization and image analysis. A 70-mer oligonucleotide microarray spanning the human genome was obtained from CapitalBio Corporation (Beijing, P.R. China). The probes provided represent 21,329 genes. The printed oligonucleotides were purchased from the Operon Company (Huntsville, AL). Labeled samples and the reference pool were hybridized overnight to the microarray at $42^{\circ} \mathrm{C}$, unbound probes were removed with stringent washing. Arrays were scanned with a LuxScan 10K/A confocal scanner (CapitalBio Corporation), and the images were transformed into digital data using LuxScan 3.0 image analysis software (CapitalBio Corporation). 
A

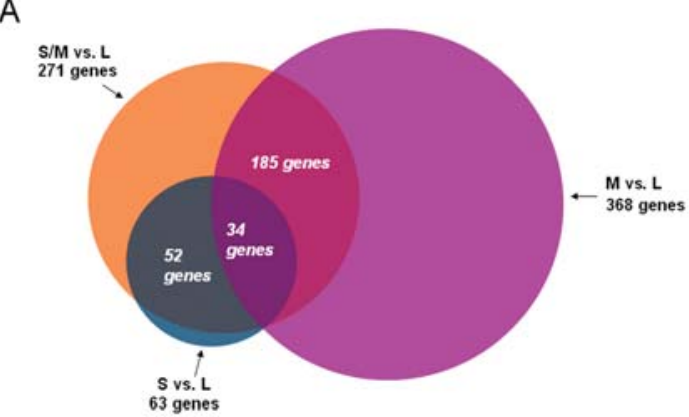

C

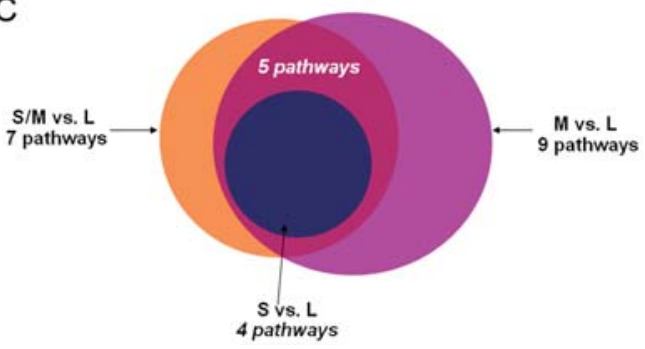

B

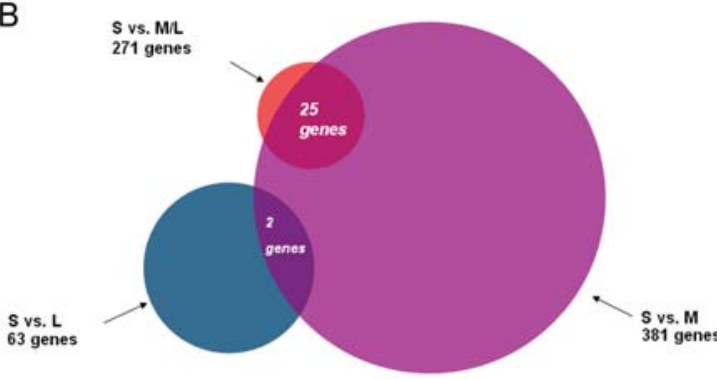

D

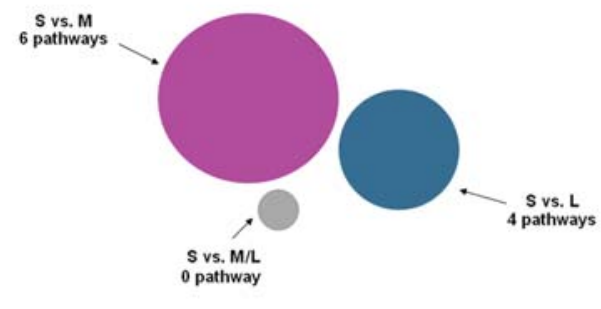

Figure 1. Overlap of genes and pathways. (A) Overlap of S/M vs. L differential genes with $\mathrm{S}$ vs. L differential genes and $\mathrm{M}$ vs. L differential genes. (B) Overlap of S vs. M/L differential genes with S vs. M differential genes and S vs. L differential genes. (C) Overlap of S/M vs. L differential pathways with $\mathrm{S}$ vs. L differential pathways and M vs. L differential pathways. (D) overlap of S vs. M/L differential pathways with S vs. M differential pathways and $\mathrm{S}$ vs. L differential pathways.

Data analysis. Microarray data were normalized using a LOWEISS normalization of each array with the $\mathrm{R}$ package. Genes with a detectable expression level in at least 9 samples were included resulting in a total of 12,660 genes in the subsequent analysis.

Unsupervised clustering analysis of the expression pattern was performed using Cluster 3.0. Treeview software was used to visualize the unsupervised clustering result. Significance analysis of microarray (SAM) by SAM software version 3.01 developed by Stanford University (7) was used for selection of differentially expressed (DE) genes. To gain insights into the cellular functions of these DE genes, the online DAVID tool (8) (http://niaid.abcc.ncifcrf. gov/) was used to mine enriched biological pathways and GO terms in DE genes. Pathway Miner in BioRag (Bioresource for array genes) at www.biorag.org was used to draw gene association networks of DE genes.

$Q R T-P C R$. Total RNA prepared for the microarray analysis was also used for quantitative real-time PCR (qRT-PCR) analysis to validate the expression level of differentially expressed genes. The RNA was reverse transcribed to cDNA and real-time PCR products were generated in 35 cycles from cDNA in a Rotor-Gene 3000 real-time PCR apparatus (Corbett Research) in a mixture containing SYBR-Green (Molecular Probes) and $1 \mu \mathrm{l}$ of $100 \mu \mathrm{M}$ specific primers for differentially expressed genes (CIITA, HLA DRA, HLA DPA1, and HLA DQB1). SYBR-Green fluorescent signals were used to generate cycle threshold values from which mRNA ratios were calculated when normalized against the GAPDH housekeeping gene.

Western blot analysis and immunohistochemistry. Total protein $(50 \mu \mathrm{g})$ from $\mathrm{HCC}$ tissue that was used for micro- array analysis was also used for Western blot analysis. Goat polyclonal anti-CIITA antibody (dilution 1:1000, Santa Cruz Biotechnology, Santa Cruz, CA) was used to detect CIITA. Rabbit polyclonal antibody $\beta$-actin (dilution 1:1000, Santa Cruz Biotechnology) was used to detect $B$-actin as a loading control.

Tumor and adjacent non-tumor tissues were obtained from 26 patients, independent of patients used for expression profiling, and were used to validate the expression of different genes on the protein level. Immunohistochemistry (IHC) staining was performed on sections of paraffin-embedded tissue samples. Anti-human HLA-DP, DQ, and DR antibodies (DakoCytomation, Carpinteria, CA) was used to detect the expression of HLA DP, DQ, and DR molecules within both tumor and non-tumor samples. Anti-human CD45 antibody (DakoCytomation) was used to stain leukocytes. Anti-human CD68 antibody (Zymed, San Francisco, CA) was used to stain Kuffer cells. Anti-human SMA antibody (Sigma, St. Louis, USA) was used to detect activated hepatic stellate cells (HSCs).

\section{Results}

Unsupervised clustering analysis. To present the similarities and differences in gene expression patterns from all 32 study participants for visual display and for subsequent analysis, an unsupervised hierarchical clustering algorithm (complete linkage, uncentered correlation) was employed. Most patients with similar tumor-free survival times were clustered together with some exceptions (data not shown).

DE genes and pathways. We identified $381 \mathrm{DE}$ genes and 6 DE pathways between S vs. M groups, $368 \mathrm{DE}$ genes and 9 DE pathways between M vs. L groups, and $63 \mathrm{DE}$ genes and 4 DE pathways between S vs. L groups (Fig. 1 and Table II). 
Table II. Summary of differential genes and pathways identified.

\begin{tabular}{|c|c|}
\hline Comparison & FDR $(\%)$ \\
\hline
\end{tabular}

S/M vs. L 17.81

271

S vs. M

18.84

19.6

368

M vs. L

S vs. L

18.03
31

271

381

$+2$
Antigen processing and presentation
Type I diabetes mellitus
Cell adhesion molecules (CAMs)
Leukocyte transendothelial migration
Natural killer cell mediated cytotoxicity
Hematopoietic cell lineage
$\mathrm{T}$ cell receptor signaling pathway

0

Colorectal cancer

Basal cell carcinoma

Endometrial cancer

Wnt signaling pathway

Melanoma

Chronic myeloid leukemia

Type I diabetes mellitus

Antigen processing and presentation

Cell adhesion molecules (CAMs)

Natural killer cell mediated cytotoxicity

Cytokine-cytokine receptor interaction

Hematopoietic cell lineage

B cell receptor signaling pathway

Leukocyte transendothelial migration

Chronic myeloid leukemia

Antigen processing and presentation

Type I diabetes mellitus

Cell adhesion molecules (CAMs)

Hematopoietic cell lineage

$\mathrm{S} / \mathrm{M}$ vs. L, the comparison between the $\mathrm{S} / \mathrm{M}$ groups and the $\mathrm{L}$ group; $\mathrm{S}$ vs. M/L, the comparison between the $\mathrm{S}$ group and $\mathrm{M} / \mathrm{L}$ groups; $\mathrm{S}$ vs. $\mathrm{M}$, the comparison between $\mathrm{S}$ and $\mathrm{M}$ group; $\mathrm{M}$ vs. L, the comparison between $\mathrm{M}$ group and $\mathrm{L}$ group; $\mathrm{S}$ vs. $\mathrm{L}$, the comparison between $\mathrm{S}$ and L group.

We also found that the $\mathrm{S}$ and $\mathrm{M}$ groups have $34 \mathrm{DE}$ genes and $4 \mathrm{DE}$ pathways in common when compared to the $\mathrm{L}$ group, while the $\mathrm{M}$ and $\mathrm{L}$ groups have only $2 \mathrm{DE}$ genes and 0 DE pathways in common when compared to the $S$ group (Fig. 1). These data indicate that early recurrence of HCC in patients of the $\mathrm{S}$ and $\mathrm{M}$ groups may be caused by similar mechanisms, and that the mechanisms involved in tumor recurrence may be identified by contrasting gene expression profiles of groups $\mathrm{S}$ and $\mathrm{M}$ with group $\mathrm{L}$.

Statistical analysis of DE genes between the S/M groups and the L group identified 271 genes, 269 of which had higher expression in group L vs. 2 genes that had higher expression in both the $\mathrm{S}$ and $\mathrm{M}$ groups (Fig. 2). Seven signaling pathways were more highly expressed in the $\mathrm{L}$ group, including proteins involved in antigen processing and presentation, Natural killer cell mediated cytotoxicity, $\mathrm{T}$ cell receptor signaling, and leukocyte transendothelial migration (Table II). The most significant differentially expressed pathway was the MHC II-related antigen processing and presentation pathway. Fifteen genes in this pathway were highly expressed in L group patients, including HLA-DPA, DQB, DRA, and HLA II gene transactivator (CIITA) (Fig. 2). These genes have also been shown to be involved in signaling by cell adhesion molecules (CAMs) and Type I diabetes mellitus pathways. Although signaling for Type I diabetes primarily takes place in the pancreas, this pathway is identified as a differential pathway because the genes involved overlap with genes in antigen processing and presentation pathways, or in CAM-dependent pathways. The GO enrichment analysis also indicated that the most enriched biological process of DE genes between S vs. L groups, M vs. L group and S/M vs. L group were the immune system process, immune response, leukocyte activation, antigen processing and presentation. Additionally, we found that expression of MHC II molecules significantly correlated with the expression level of most immune related DE genes between the S/M and L groups (data not shown), as well as with the expression level of CIITA. 


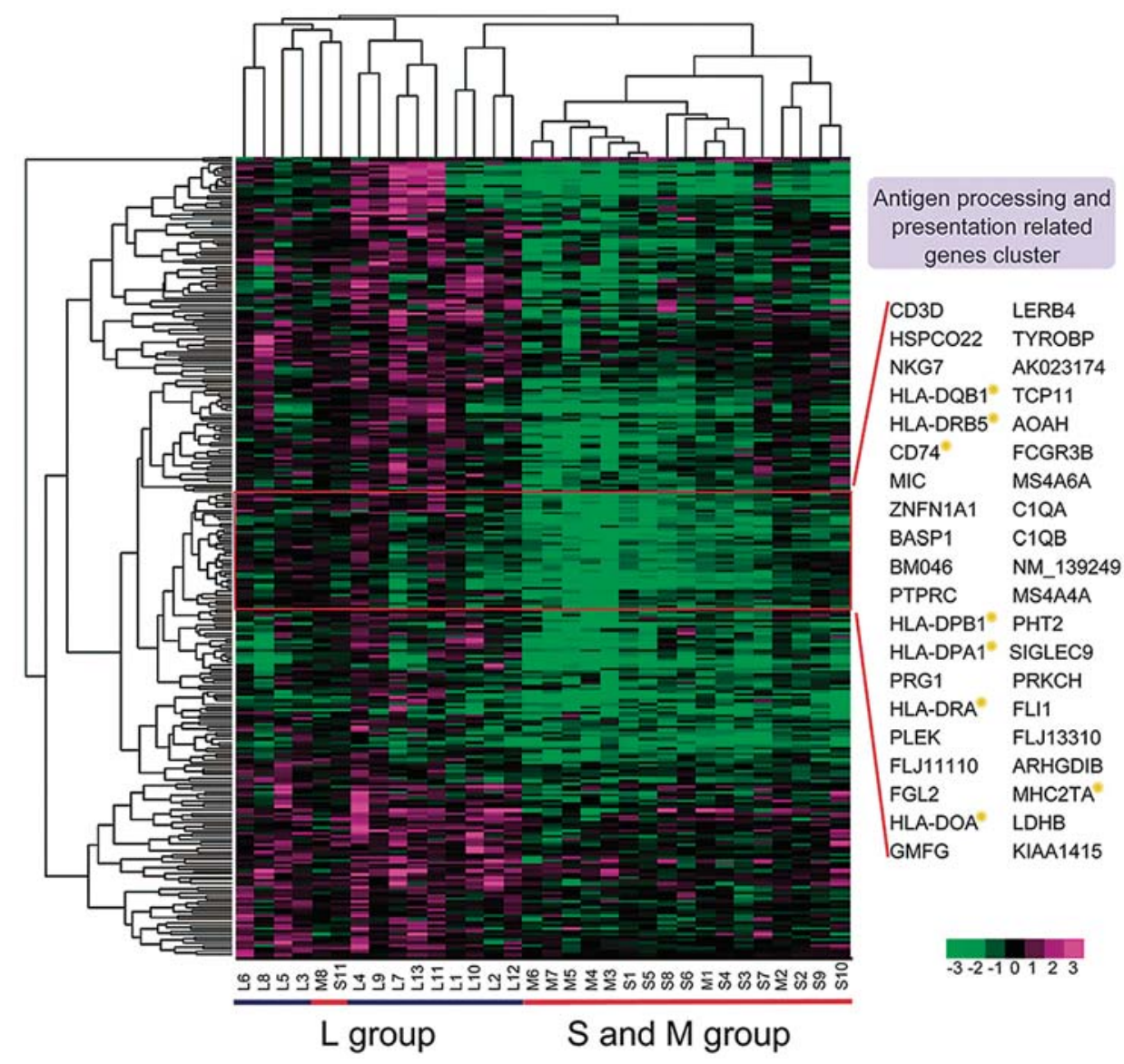

Figure 2. Clustering analysis of differential genes. Clustering of 271 genes differentially expressed between the combination of S and M samples vs. group L samples. The genes listed in this figure belong to an antigen processing and presentation gene cluster, and the genes belonging to antigen processing and presentation pathway are marked with a star.

A

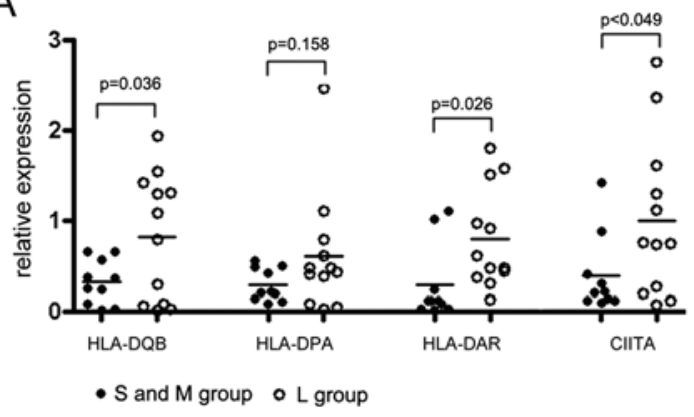

$\mathrm{B}$

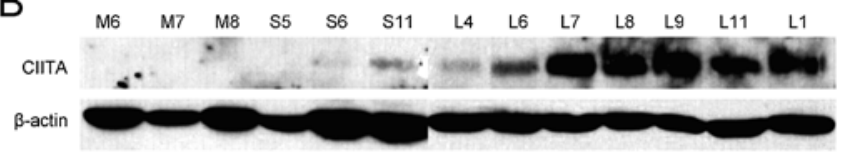

C

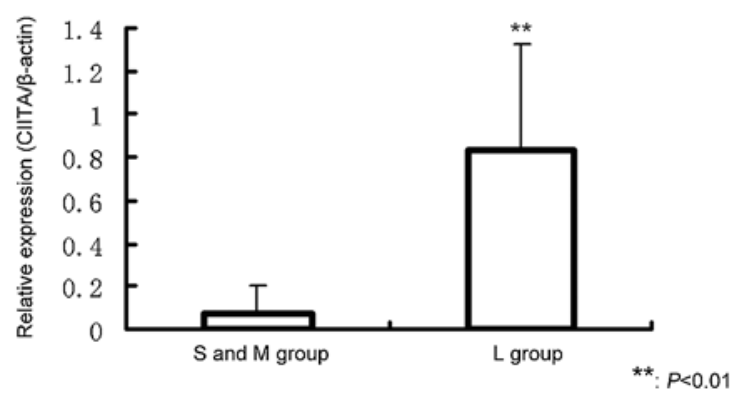

Figure 3. Expression of HLA gene and CIITA. (A) qRT-PCR validation of HLA and CIITA in HCC tumor tissue. Relative expression fold of each gene normalized to GAPDH and a non-tumor adjacent tissue pool is shown for HLA DR, DP, DQ and CIITA. (B and C) Expression of CIITA in S and M grouped sample vs. L group samples detected by Western blot. Expression levels differ significantly between these two groups of patients.

Expression of CIITA and HLA DR,DP and DQ. The expression levels of HLA DR, DP, DQ, and CIITA in HCC patients were detected using qRT-PCR and were found to be consistent with the expression patterns detected in the microarray analysis (Fig. 3A). Differential expression of HLA DR, DQ, and CIITA was significant between S/M and
L group. But statistical significance could not be determined for the expression level of HLA DP between S/M groups and the L group due to limited sample numbers. Overall, the expression of CIITA significantly correlated with the expression level of HLA DR, DP, and DQ by qRT-PCR data (data not shown). The expression level of CIITA in S/M 
A

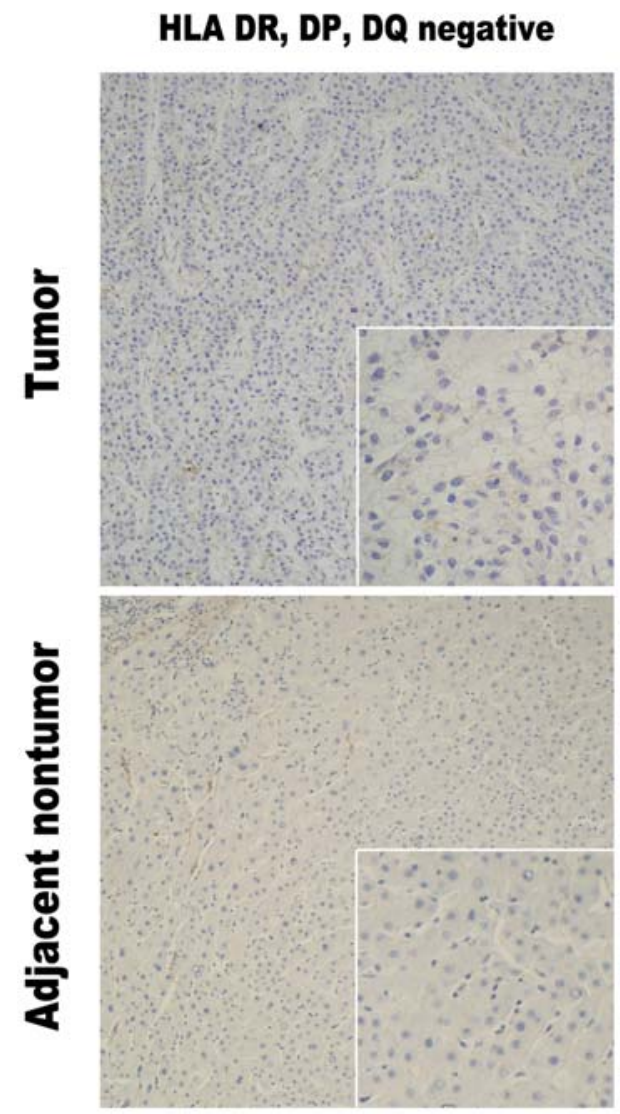

B

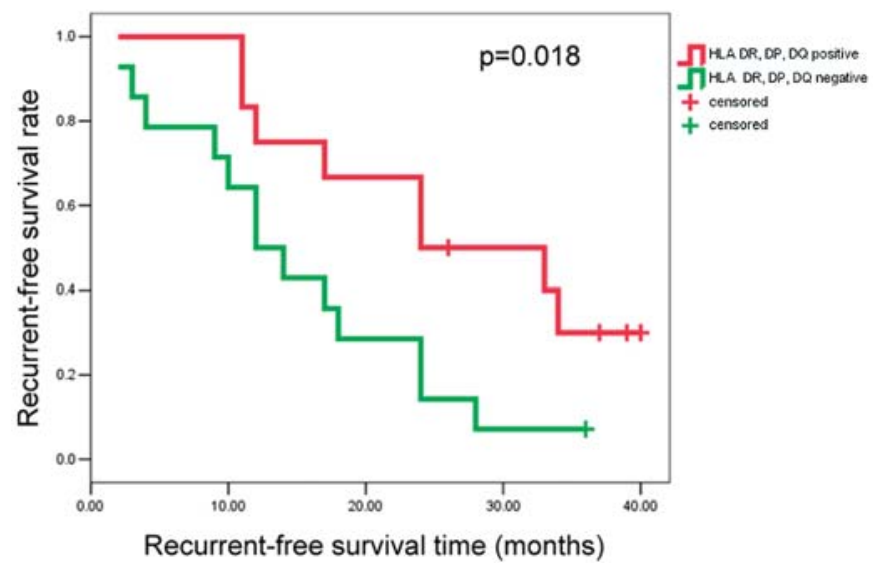

and $\mathrm{L}$ group samples was also validated by Western blot and was found to be significantly different between patients with and without $\mathrm{HCC}$ recurrence within 3 years $(\mathrm{p}<0.001)$ (Fig. 3B and C).

IHC analysis of 26 paraffin-embedded patient tissue samples, independent of samples used in the microarray analysis, demonstrated that protein expression of HLA DR, DP and DQ was significantly different between patients recurrent or not within 3 years after resection $(0.026$ by Fisher's exact test). Five out of six HCC patients with an RFS time $>3$ years expressed HLA DR, DP, and DQ within the tumor tissue (Fig. 4A). We further determined that the RFS time between HLA DR/DP/DQ ${ }^{+}$and HLA DR/DP/DQpatients was significantly different (Fig. 4B).

\section{HLA DR, DP, DQ positive}
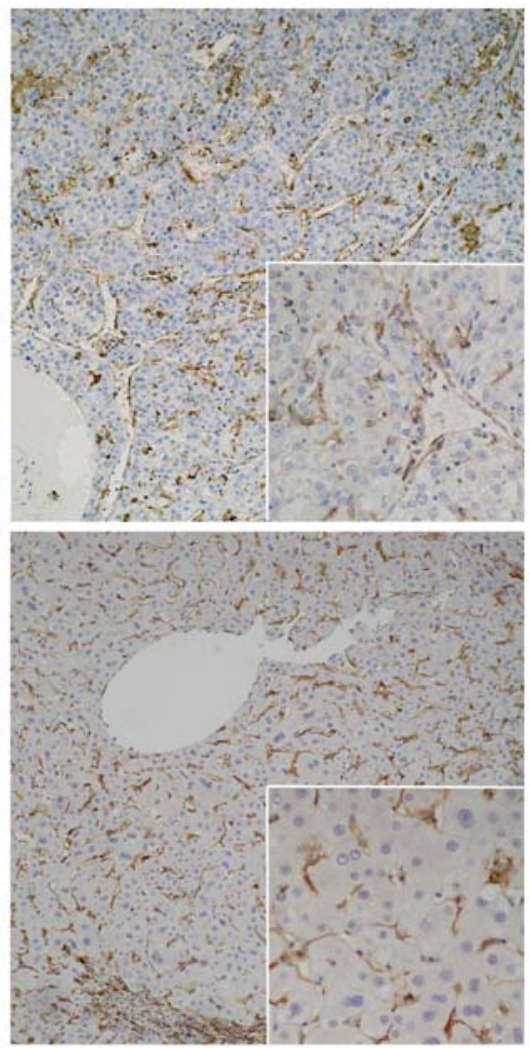

Figure 4. IHC analysis of HLA DR, DP and DQ protein expression in $\mathrm{HCC}$ tumor and adjacent non-tumor tissue samples. (A) Expression and localization of HLA DR, DP, and DQ proteins in HCC tumor and adjacent non-tumor tissue samples. (B) Survival analysis of the RFS time of HCC patients with or without HLA DR, DP and DQ protein expression in the tumor.

Most HLA DR/DP/DQ ${ }^{+}$cells within the tumor localized in the sinusoid and just a small part of HLA DR/DP/DQ ${ }^{+}$ cells overlapped with the tumor cells (Fig. 4A). Most HLA $\mathrm{DR} / \mathrm{DP} / \mathrm{DQ}^{+}$cells appeared to overlap with either $\mathrm{CD}^{+} 5^{+}$cells or $\mathrm{CD} 68^{+}$cells, especially $\mathrm{CD} 45^{+}$cells, but no HLA DR/ $\mathrm{DP} / \mathrm{DQ}^{+}$cells overlapped with $\mathrm{SMA}^{+}$cells (data no shown). These results suggest that $\mathrm{HLA} \mathrm{DR} / \mathrm{DP} / \mathrm{DQ}^{+}$cells are mainly contributed by cells in the liver sinusoid, including Kuffer cells and CD45 leukocytes, and partially by tumor cells. It was also observed that samples derived from adjacent nontumor tissue of HCC patients with HLA DR/DP/DQ ${ }^{+}$cells, also have strong HLA DR/DP/DQ expression. These HLA $\mathrm{DR} / \mathrm{DP} / \mathrm{DQ}^{+}$cells were all located in the liver sinusoid and overlapped with either CD68 ${ }^{+}$cells or $\mathrm{CD} 45^{+}$cells (Fig. 4A). However, the expression of HLA DR/DP/DQ was not always simultaneously expressed in both tumor and adjacent nontumor tissue samples.

\section{Discussion}

We compared the clinical factors of these 3 groups of patients, and significant differences were only found in their tumor stages determined by AJCC TNM tumor staging system (Table I). However, a comparison of the RFS time between TNM stage I/II vs. TNM stage III/IV HCC patients was not significant $(\mathrm{p}=0.326)$, but our unsupervised 


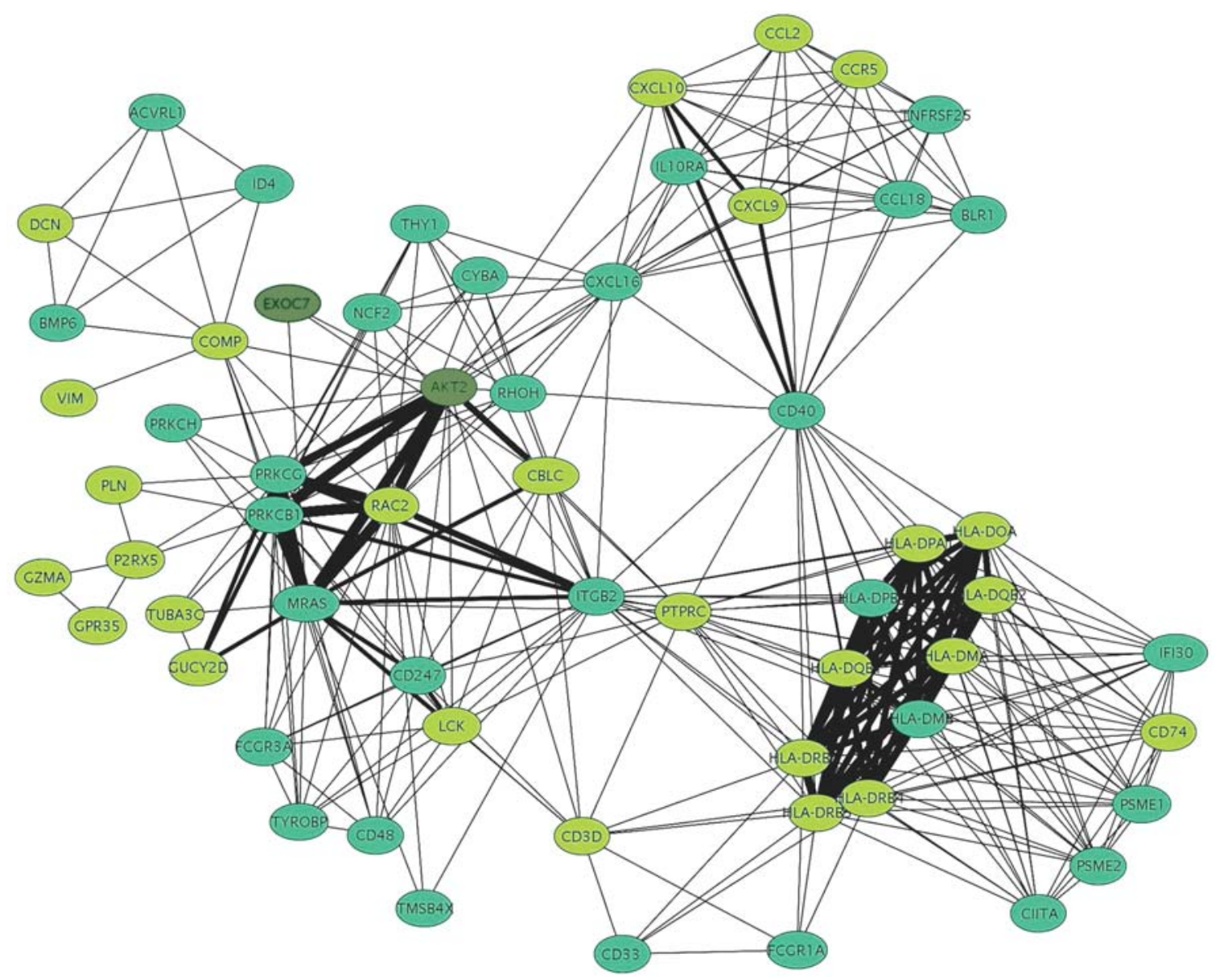

Figure 5. The association network of SM vs. L differential genes. This network was constructed using Pathway Miner from the Biorag website. Each spot represents a gene highly expressed in the L group. The thickness of edges between two genes indicates the number of pathways that could be associated with these two genes.

clustering data showed that HCC patients with different RFS time following surgical resection do have distinguishable gene expression patterns, and this result is consistent with similar studies published in recent years (2-4), and provide additional insight into our understanding of the mechanisms involved in the recurrence of HCC.

Our data suggest that recurrence of HCC within 3 years of surgical resection (i.e. $\mathrm{S}$ and $\mathrm{M}$ group patients) may be caused by some similar mechanism when compared with those patients with an RFS time larger than 3 years. While previous studies (2-4,9-12) have investigated differential gene expression patterns between patients with or without recurrence/metastasis within 1 year after surgery, which may fail to unfold this phenomenon. The DE gene and pathway analysis indicated that many immune related genes and pathways have a lower expression level in patients with an RFS time $<3$ years. The GO enrichment analysis showed similar result that most DE genes between $\mathrm{S} / \mathrm{M}$ and L group were involved in the immune process. The association of these DE genes also indicates an immune-related interaction network, and predicts that the anti-tumor immune response is an important factor in the prognosis of HCC patients following surgical resection (Fig. 5). Therefore, we hypothesize that patients with strong anti-tumor immune responses in tumor microenvironment may have longer RFS time, which is supported by the data in this study and others $(10,13,14)$.
MHC II genes play a vital role in the immune response, including antigen presentation and immune activation. Antigen processing and presentation pathways showed the most significant differences in gene expression between $\mathrm{S} / \mathrm{M}$ vs. L groups. HLA DR, DP, and DQ were the genes found to significantly correlate with the expression levels of many immune-related genes. The important role that MHC II genes play in the immune response, and the significant correlation between MHC II genes and most immune-related genes in HCC patients, suggests that expression levels of MHC II genes play a role in anti-tumor immune responses of HCC patients. This may explain why HCC patients with higher expression of MHC II genes have longer RFS time. This result partly agree with previous studies that have identified one or more MHC II molecules associated with the prognosis of HCC $(14,15-18)$.

Our data indicate that these highly expressed MHC II molecules in HCC tumors were primarily contributed by cells in the liver sinusoid, including $\mathrm{CD} 68^{+}$cell and CD45+ leukocytes. This finding strongly indicated that the infiltrated immune cell type, number and function in tumor immune microenvironment is one of the most important roles that determine the post-surgery RFS time. We also found that samples derived from adjacent non-tumor tissue of HCC patients with HLA DR/DP/DQ ${ }^{+}$cells, also have strong HLA DR/DP/DQ expression. Therefore, additional studies are 
needed to identify the mechanistic details of the role of MHC II molecules in the prognosis of HCC patients.

CIITA, a transactivator of HLA II genes, is a mediator between the MHC-enhanceosome and the gene transcription initiation machinery. CIITA is essential for transcriptional activation of all HLA II genes $(19,20)$. Correspondingly, CIITA was found to be differentially expressed between $\mathrm{S} / \mathrm{M}$ vs. L group samples and significantly correlated with the expression level of HLA II genes (Fig. 3). We hypothesize that changes in expression of HLA II genes in HCC patients is related to CIITA expression levels. In gastrointestinal cancer cells and other types of tumors, the relationship between CIITA and HLA II gene expression has been associated with patient prognosis (21-27). These studies found that loss of CIITA expression contributed to the down-regulation of HLA II genes in tumors and lead to the immune escape of tumor cells. Our study is the first to look at these processes in HCC samples and to identify CIITA as a possible target for therapy and prevention of recurrence in HCC.

In conclusion, our data highlight the importance of the expression level of CIITA and MHCII molecules, which may be effective prognostic markers for HCC. HCC patients with higher CIITA expression in tumor tend to have higher MHC II genes and immune-related genes expression level, and are more likely to have longer recurrent-free survival (RFS) time. We also proved that these MHCII molecules are mainly located in either $\mathrm{CD} 68^{+}$cells or $\mathrm{CD} 45^{+}$cells, demonstrating that the infiltrated immune cell type, number and function in tumor immune microenvironment is one of the most important roles that determine the post-surgery RFS time.

\section{Acknowledgments}

This work was supported by the National Natural Science Foundation of China (30772499), National Key Sci-Tech Special Project of China (no. 2008ZX10002-019), Program for New Century Excellent Talents (985-2-099-113) and Guangxi Scientific Research and Technological Development Project (0428005-7). GEO accession numbers: microarray platform, GPL4525; microarray data, GSE11260.

\section{References}

1. Tang ZY, Ye SL, Liu YK, et al: A decade's studies on metastasis of hepatocellular carcinoma. J Cancer Res Clin Oncol 130: 187-196, 2004

2. Lee JS and Thorgeirsson SS: Genome-scale profiling of gene expression in hepatocellular carcinoma: classification, survival prediction and identification of therapeutic targets. Gastroenterology 127: 51-55, 2004

3. Lee JS, Chu IS, Heo J, et al: Classification and prediction of survival in hepatocellular carcinoma by gene expression profiling. Hepatology 40: 667-676, 2004.

4. Ye QH, Qin LX, Forgues M, et al: Predicting hepatitis B virus-positive metastatic hepatocellular carcinomas using gene expression profiling and supervised machine learning. Nat Med 9: 416-423, 2003.

5. Iizuka N, Oka M, Yamada-Okabe H, et al: Oligonucleotide microarray for prediction of early intrahepatic recurrence of hepatocellular carcinoma after curative resection. Lancet 361: 923-929, 2003

6. Patterson TA, Lobenhofer EK, Fulmer-Smentek SB, et al: Performance comparison of one-color and two-color platforms within the MicroArray Quality Control (MAQC) project. Nat Biotechnol 24: 1140-1150, 2006.
7. Tusher VG, Tibshirani R and Chu G: Significance analysis of microarrays applied to the ionizing radiation response. Proc Natl Acad Sci USA 98: 5116-5121, 2001.

8. Dennis G Jr, Sherman BT, Hosack DA, et al: DAVID: Database for annotation, visualization, and integrated discovery. Genome Biol 4: P3, 2003

9. Ho MC, Lin JJ, Chen CN, et al: A gene expression profile for vascular invasion can predict the recurrence after resection of hepatocellular carcinoma: a microarray approach. Ann Surg Oncol 13: 1474-1484, 2006

10. Budhu A, Forgues M, Ye QH, et al: Prediction of venous metastases, recurrence, and prognosis in hepatocellular carcinoma based on a unique immune response signature of the liver microenvironment. Cancer Cell 10: 99-111, 2006.

11. Iizuka N, Oka M, Yamada-Okabe H, et al: Comparison of gene expression profiles between hepatitis B virus- and hepatitis C virus-infected hepatocellular carcinoma by oligonucleotide microarray data on the basis of a supervised learning method. Cancer Res 62: 3939-3944, 2002.

12. Kurokawa Y, Matoba R, Takemasa I, et al: Molecular-based prediction of early recurrence in hepatocellular carcinoma. J Hepatol 41: 284-291, 2004.

13. Ohira M, Ohdan H, Mitsuta H, et al: Adoptive transfer of TRAILexpressing natural killer cells prevents recurrence of hepatocellular carcinoma after partial hepatectomy. Transplantation 82: 1712-1719, 2006

14. Takayama T, Sekine T, Makuuchi M, et al: Adoptive immunotherapy to lower postsurgical recurrence rates of hepatocellular carcinoma: a randomised trial. Lancet 356: 802-807, 2000.

15. Shirabe K, Matsumata T, Maeda T, Sadanaga N, Kuwano H and Sugimachi K: A long-term surviving patient with hepatocellular carcinoma including lymphocytes infiltration: a clinicopathological study. Hepatogastroenterology 42: 996-1001, 1995.

16. Schoniger-Hekele M, Hanel S, Wrba F and Muller C: Hepatocellular carcinoma - survival and clinical characteristics in relation to various histologic molecular markers in Western patients. Liver Int 25: 62-69, 2005

17. Reinisch W, Holub M, Katz A, et al: Prospective pilot study of recombinant granulocyte-macrophage colony-stimulating factor and interferon-gamma in patients with inoperable hepatocellular carcinoma. J Immunother 25: 489-499, 2002.

18. Matoba K, Iizuka N, Gondo T, et al: Tumor HLA-DR expression linked to early intrahepatic recurrence of hepatocellular carcinoma. Int J Cancer 115: 231-240, 2005.

19. Wright KL and Ting JP: Epigenetic regulation of MHC-II and CIITA genes. Trends Immunol 27: 405-412, 2006.

20. Holling TM, van EM, Jager MJ and van EP: Epigenetic silencing of MHC2TA transcription in cancer. Biochem Pharmacol 72: 1570-1576, 2006

21. Shi B, Vinyals A, Alia P, et al: Differential expression of MHC class II molecules in highly metastatic breast cancer cells is mediated by the regulation of the CIITA transcription implication of CIITA in tumor and metastasis development. Int J Biochem Cell Biol 38: 544-562, 2006.

22. Morimoto Y, Toyota M, Satoh A, et al: Inactivation of class II transactivator by DNA methylation and histone deacetylation associated with absence of HLA-DR induction by interferongamma in haematopoietic tumour cells. Br J Cancer 90: 844-852, 2004.

23. Hippo Y, Yashiro M, Ishii M, et al: Differential gene expression profiles of scirrhous gastric cancer cells with high metastatic potential to peritoneum or lymph nodes. Cancer Res 61: 889-895, 2001.

24. Boye K, Andersen K, Tveito S, Oyjord T and Maelandsmo GM: Interferon-gamma-induced suppression of S100A4 transcription is mediated by the class II transactivator. Tumour Biol 28: 27-35, 2007.

25. Van SN, Quinten E, Alblas G, et al: Constitutive and IFNgamma-induced activation of MHC2TA promoter type III in human melanoma cell lines is governed by separate regulatory elements within the PIII upstream regulatory region. Mol Immunol 44: 2036-2046, 2007.

26. Satoh A, Toyota M, Ikeda H, et al: Epigenetic inactivation of class II transactivator (CIITA) is associated with the absence of interferon-gamma-induced HLA-DR expression in colorectal and gastric cancer cells. Oncogene 23: 8876-8886, 2004.

27. Iizuka $\mathrm{N}$ and Oka M: CIITA methylation and decreased levels of HLA-DR in tumour progression. Br J Cancer 91: 813814-813815, 2004. 